\title{
Leptospirosis en la población de la Región Huetar Norte durante el periodo comprendido entre 1997 y 2000.
}

Ana Catalina Sáenz -Zamora, Gilberto Rodríguez -Herrera

\section{R e sume n}

Servicio de Emergencias, Hospital de San Carlos. Alajuela, Costa Rica.

Abreviaturas: ADN, Ácido desoxirribonucleico; AST, aspartato aminotransferasas; CPK, creatinin fosfoquinasa; ELISA, siglas en inglés de enzyme-linked inmunosorbent assay; HSC, Hospital de San Carlos; INCIENSA, Instituto Costarricense de Investigación y Enseñanza en Nutrición y Salud; MAT, test de microaglutinación; PCR, reacción en cadena de polimerasa.

Correspondencia: Ana Catalina Sáenz Zamora

E-mail: catsaenz@costarricense.cr

ISSN 0001-6002/2004/46/4/184-189 Acta Médica Costarricense, (C2004 Colegio de Médicos y Cirujanos
Justificación y objetivo: Determinar el comportamiento epidemiológico de la leptospirosis en la población de la Región Huetar Norte, durante el periodo comprendido entre 1997 y 2000, según la información biomédica de la sede regional del Ministerio de Salud y del Hospital San Carlos.

Método: Se pidió a INCIENSA una lista de los casos con serología positiva por leptospirosis de la Región Huetar Norte, vistos de 1997 al 2000; se solicitaron los expedientes clínicos de estos pacientes a la oficina de Bioestadística del Hospital San Carlos y de allí se obtuvieron los datos requeridos por una hoja previamente confeccionada.

Resultados: Se encontró un total de 58 pacientes diagnosticados con leptospirosis en dicha región, en el periodo en estudio; predominó el género masculino, en una relación de 13:1. La población más afectada fue la de los adultos jóvenes, entre los 15 y los 30 años. El cantón de donde provinieron más personas con la enfermedad fue San Carlos. Más de la tercera parte de estas personas se ocupaban de labores agrícolas o ganadería. Los serotipos encontrados de manera más frecuente fueron pyrogenes, seguido de sejroe, tarassovi y hebdomadis. Las principales manifestaciones fueron: fiebre, cefalea, mialgias, sangrados, alteración en las pruebas de función renal y hepática, trombocitopenia y aumento de la creatinin fosfoquinasa.

Conclusiones: En casi la totalidad de los pacientes fue posible determinar factores de riesgo, y en la mayoría el principal factor fue la exposición ocupacional. El hallazgo de leucopenia es tres veces más frecuente que el de leucocitosis, por lo que se puede afirmar que existe un comportamiento clínico diferente de la leptospirosis descrito en la bibliografía.

Descriptores: zoonosis, espiroqueta, enfermedad de Weil, leptospirosis.

Recibido: 16 de diciembre de 2003

Aceptado: 20 de julio de 2004

La leptospirosis es una zoonosis de distribución mundial, producida por espiroquetas del género Leptospira, que afecta a gran variedad de animales salvajes y domésticos. El hombre se contagia al entrar en contacto con animales infectados o con el medio contaminado ${ }^{1}$.

La familia Lestospiraceae pertenece al orden Spirochaetales, y se divide en tres géneros Leptospira, Leptonema y Turneria. Leptospira se ha subdividido en 250 serovares por medio de la prueba de microaglutinación. Los serovares se clasifican en grupos serológicos por la presencia de antígenos comunes. Taxonómicamente leptospira se clasifica en 12 especies, que se ordenan en tres grupos: patógenas, intermedios y saprófitos. La taxonomía molecular está en progreso y va a sustituir eventualmente al sistema de serovares. 
Manifestaciones clínicas específicas y gravedad de la enfermedad no se asocian con serovares específicos. La división en serogrupos tiene importancia epidemiológica, porque permite identificar fuentes comunes en brotes epidémicos ${ }^{2,3}$.

Leptospira es una espiroqueta aeróbica con una membrana citoplasmática y pared celular, rodeadas por una membrana exterior ${ }^{3}$.

La mayoría de los casos en América Latina ocurren en hombres adultos (88\% de los enfermos), principalmente durante la cuarta o quinta década de la vida, en poblaciones rurales y en los meses de lluvia ${ }^{4,5}$. A pesar de la distribución mundial de la leptospirosis, son las regiones tropicales las que sobrellevan el mayor impacto, más aún en los lugares donde la población vive en contacto estrecho con animales ${ }^{2,6}$.

Si bien a nivel mundial las ratas constituyen la fuente más importante de infección humana, en nuestro país se cree que este lugar lo ocupa el ganado bovino y los perros, pero, no existe consenso sobre este tema ${ }^{3}$.

Las infecciones por exposición ocupacional (veterinarios, ganaderos, carniceros, agricultores) y por exposición recreativa (nadadores, triatlonistas) son cada vez más frecuentes ${ }^{3,7}$.

En Costa Rica, el primer caso fue registrado a inicios de la década de los ochenta en la Península de Osa. En 1988 se presentó un brote epidémico en la provincia de Puntarenas, con 81 casos y 4 defunciones. Posteriormente, ha habido casos esporádicos, en su mayoría en la Meseta Central, de personas que van a vacacionar a las costas o montañas y tienen contacto con aguas contaminadas. La región Brunca ocupa el primer lugar a nivel nacional en incidencia, reportando casi la tercera parte de los casos (370 pacientes entre 1997 y 2000), y la región Huetar Norte ocupa el tercero, con cerca del $11 \%$. Los serotipos hallados con más frecuencia en la infección humana, en nuestro país, son: icterohaemorrhagiae, australis, hardjo, pyrogenes, canicola, pomona, sejroe y bataviae ${ }^{2,3,8,9}$.

El periodo de incubación de la leptospirosis oscila entre 2 y 30 días, siendo en promedio 5-12 días. Todas las presentaciones clínicas de esta enfermedad empiezan de igual manera e inicialmente es imposible saber si se trata de una forma leve o grave de leptospirosis, porque la lesión inicial puede estar influenciada, entre otros factores, por la virulencia del serovar infectante, la magnitud del inóculo, el estado nutricional del paciente y el tiempo que se tarde en iniciar el tratamiento adecuado ${ }^{1-3}$.

Alrededor del 15\% de las infecciones cursan de forma subclínica, solo evidenciadas por una seroconversión. Entre las personas que desarrollan manifestaciones clínicas, el $90 \%$ tiene una forma leve anictérica y el 5-10\% una forma grave, conocida como enfermedad o síndrome de Weil ${ }^{1,3,10}$. En la forma ictérica, los síntomas descritos para los pacientes anictéricos son más intensos y prolongados ${ }^{11}$.
La epistaxis y otros sangrados menores, tales como gingivorragia, petequias, hematomas y hemoptisis, son muy comunes. La hemoptisis ocurre como resultado de hemorragias alveolares e intersticiales y, junto con el dolor torácico y la tos, comprenden las manifestaciones pulmonares de la enfermedad, cuya frecuencia oscila entre el $25 \%$ y el $80 \%{ }^{2,12}$.

El diagnóstico de leptospirosis muchas veces se omite o se hace de manera tardía, debido a que los médicos no tienen la enfermedad en mente, por su similitud con el dengue y otros procesos virales, así como por la ausencia de pruebas diagnósticas en las que se obtenga un resultado rápido, o por dificultad de acceso a un laboratorio que las realice. Así, por ejemplo, en Costa Rica el único laboratorio habilitado para efectuar tales pruebas es el del Instituto Costarricense de Investigación y Enseñanza en Nutrición y Salud (INCIEN$\mathrm{SA}$ ). Además, debido a que las manifestaciones iniciales de la leptospirosis son inespecíficas, debe descartarse al mismo tiempo la posibilidad por malaria y dengue ${ }^{8,13}$.

El estándar de oro para el diagnóstico de leptospirosis es el test de microaglutinación (MAT), que mide la presencia de anticuerpos $\operatorname{IgG}^{7,14}$.

La reacción en cadena de polimerasa (PCR) detecta por amplificación, el ADN de las espiroquetas en la sangre u orina del paciente. Aunque se encuentra bajo estudio y no es accesible para uso general, la PCR promete ser una excelente opción para la detección temprana de leptospirosis. Comparando la PCR con el MAT en una muestra de 200 pacientes, varios grupos de investigadores han encontrado que la PCR detecta ADN de las leptospiras, en el suero o en la orina, entre los días 2 y 5 del inicio de la enfermedad. Estos investigadores identificaron leptospiras por medio de la PCR en 13 de 71 pacientes que habían sido identificados como negativos por el MAT ${ }^{15,16}$. En otros estudios recientes se ha demostrado que la medición de la PCR en orina tuvo mayor éxito detectando el ADN que la de suero, y que los resultados de las pruebas de PCR, fueron devueltos en 24 horas, en contraste con la medición de anticuerpos o los cultivos, cuyos reportes toman varias semanas ${ }^{17,18}$.

Un estudio publicado por la revista World Disease Weekly, muestra que la incorporación de anticuerpos monoclonales a ELISA puede usarse en un futuro no muy lejano, para la detección de anticuerpos específicos para los distintos serovares de L.interrogans ${ }^{19-20}$.

\section{Materiales y métodos}

Se realizó un estudio retrospectivo en una población que se obtuvo identificando los casos reportados por INCIENSA con serología positiva por leptospirosis, en la Región Huetár Norte. Se pidió la lista de expedientes clínicos de estos pacientes a la oficina de Bioestadística del 
Hospital San Carlos y se recolecto la información en un formulario prediseñado con las variables de interés.

Criterios de inclusión: con las variables de interés serológico por leptospirosis positiva, residencia en algunas áreas de salud de la Región Huetar Norte o sus zonas de atracción, durante el período comprendido entre el 1 de enero de 1997 y el 31 de diciembre del 2000. Se excluyeron del estudio los casos con información incompleta, ambigua o ilegible.

Las variables cuantitativas incluidas en el estudio fueron: año y mes de diagnóstico y la edad correspondiente y las variables cualitativas fueron: género, ocupación, cantón de procedencia, motivo de consulta, hallazgos de la exploración clínica, alteraciones de laboratorio, presencia de factores de riesgo y el serotipo de Leptospira con mayor titulación.

Para determinar la incidencia y la prevalencia de leptospirosis en la población de la Región Huetar Norte, se construyeron tasas por 100000 habitantes, con el número de habitantes de la región suministrado por el Instituto Nacional de Estadística y Censos ${ }^{21}$.

\section{Resultados}

La población en estudio estuvo constituida por 58 casos (54 varones y 4 mujeres). La distribución, según la edad, mostró que el $43,1 \%$ de los casos estaba entre los 15 y los 30 años (figura 1).

La mayoría de los pacientes provienen del cantón de San Carlos (93.1\%), seguido de Guatuso (5.1\%) y Los Chiles (1.7\%).

La actividad laboral con riesgo de exposición a leptospirosis se identificó en 41 personas $(70.7 \%)$. Dentro de este grupo $34.4 \%$ eran peones agrícolas, $20.6 \%$ agricultores y $15.5 \%$ ejercían una actividad laboral que los mantenía en contacto con animales o aguas estancadas.

El serotipo de la leptospirosis se identificó en 55 casos (94.8\%). En los 3 casos en que no se identificó el serotipo tenían anticuerpos tipo IgM positivos por L. Interrogans (cuadro 2).

El motivo de consulta más frecuente fue fiebre 57 casos $(98.2 \%)$. Otros síntomas fueron mialgias, cefalea, vómitos, dolor abdominal, diarrea y sangrado (cuadro 3 ).

Los principales hallazgos durante la exploración física fueron ictericia, sangrado y hepatomegalia (cuadro 4).

La distribución de las alteraciones de laboratorio se

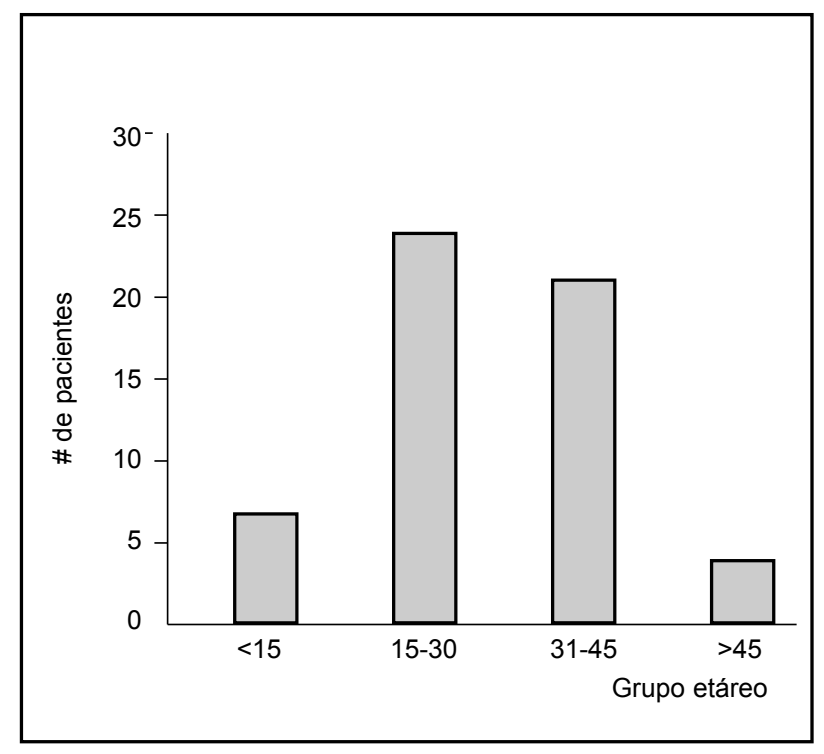

Figura 1. Distribución de la población según grupos de edad.

\begin{tabular}{|c|c|c|c|c|c|c|c|}
\hline \multicolumn{8}{|c|}{$\begin{array}{l}\text { Cuadro 1: Distribución de la población } \\
\text { según serotipo y procedencia }\end{array}$} \\
\hline \multicolumn{2}{|c|}{ Los Chiles } & \multicolumn{2}{|c|}{ Guatuso } & \multicolumn{2}{|c|}{ San Carlos } & \multicolumn{2}{|c|}{ Total } \\
\hline Serotipo & $\%$ & $\mathbf{N}$ & $\%$ & $\mathbf{N}$ & $\%$ & $\mathbf{N}$ & $\%$ \\
\hline Australis & 0 & 0 & 0 & 5 & 8.62 & 5 & 8.62 \\
\hline Bataviae & 0 & 1 & 1.72 & 3 & 5.17 & 4 & 6.89 \\
\hline Canicola & 0 & 0 & 0 & 4 & 6.89 & 4 & 6.89 \\
\hline Castellonis & 0 & 0 & 0 & 5 & 8.62 & 5 & 8.62 \\
\hline Grippotyphosa & 0 & 0 & 0 & 5 & 8.62 & 5 & 8.62 \\
\hline Hebdomadis & 0 & 1 & 1.72 & 5 & 8.62 & 6 & 10.23 \\
\hline $\begin{array}{l}\text { Icterohae- } \\
\text { morrhagiae }\end{array}$ & 0 & 0 & 0 & 3 & 5.17 & 3 & 5.17 \\
\hline Pomona & 0 & 0 & 0 & 4 & 6.89 & 4 & 6.89 \\
\hline Pyrogenes & 0 & 0 & 0 & 7 & 12.06 & 7 & 12.06 \\
\hline Sejroe & 0 & 0 & 0 & 6 & 10.34 & 6 & 10.34 \\
\hline Tarassovi & 0 & 1 & 1.72 & 5 & 8.62 & 6 & 10.34 \\
\hline No reportado & 1.72 & 0 & 0 & 2 & 3.44 & 3 & 5.17 \\
\hline TOTAL & 1.72 & 3 & 5.17 & 54 & 93.1 & 58 & 100 \\
\hline
\end{tabular}

describe en el cuadro 5. Los hallazgos de laboratorio mas frecuentes fueron: trombocitopenia, anormalidad en las pruebas de función hepática, leucopenia y aumento de la CPK en 32 casos. En los pacientes con función hepática anormal, el 97,5\% (39 casos) presentó aumento en los niveles séricos de bilirrubinas (con predominio de la bilirrubina conjugada) y de las transaminasas (principalmente AST). En el restante $2,5 \%$ solo se registró aumento de transaminasas. 


\begin{tabular}{|lcc|}
\hline \multicolumn{2}{|c|}{$\begin{array}{c}\text { Cuadro 2: Serotipos identificados en } \\
55 \text { casos de leptospirosis. }\end{array}$} \\
\hline Serotipo & $\mathrm{N}$ & $\%$ \\
\hline Pyrogenes & 7 & 13 \\
Sejroe & 6 & 11 \\
Tarassovi & 6 & 11 \\
Hebdomadis & 6 & 11 \\
Australis & 5 & 9 \\
Castellonis & 5 & 9 \\
Grippotyphosa & 5 & 9 \\
Pomona & 4 & 7 \\
Bataviae & 4 & 7 \\
Canicola & 4 & 7 \\
Icterohaemorrhagiae & 3 & 6 \\
\hline
\end{tabular}

\begin{tabular}{|lcc|}
\hline \multicolumn{3}{|c|}{$\begin{array}{c}\text { Cuadro 3: Síntomas más frecuentes } \\
\text { como motivo de consulta en } \\
\text { de leptospirosis } \\
\text { casos }\end{array}$} \\
\hline Síntoma & $\mathrm{N}$ & $\%$ \\
\hline Fiebre & 57 & 98.3 \\
Mialgias & 39 & 67.2 \\
Cefalea & 36 & 62.1 \\
Vómitos & 33 & 56.8 \\
Dolor abdominal & 22 & 37.9 \\
Diarrea & 18 & 31.0 \\
Sangrado & 7 & 12.1 \\
\hline
\end{tabular}

\section{Discusión}

Los resultados del análisis epidemiológico realizado a la población en estudio revelaron que la relación de hombres con respecto a las mujeres (13:1), coincide con la bibliografía revisada. Así, por ejemplo, el Centro para el Control y Prevención de Enfermedades de Estados Unidos reportó una incidencia del $88 \%$ en el masculino y un $12 \%$ en el femenino ${ }^{5,22}$. La mayor frecuencia de leptospirosis en el género masculino en la Región Huetar Norte, puede basarse en el hecho de que son los hombres quienes están más expuestos a los factores de riesgo ocupacionales mencionados en este estudio.

Los datos encontrados con respecto a edad difieren de otros estudios revisados, por ejemplo, Torres encontró que la incidencia de leptospirosis tiende a aumentar después de los 30 años ${ }^{2}$. Los datos encontrados en la Región Huetar Norte se explican porque, de acuerdo con el Instituto Nacional de Estadística y Censos, las personas menores de

\section{Cuadro 4: Hallazgos al exámen físico inicial en} 58 casos de leptospirosis

\begin{tabular}{|lcc|}
\hline Hallazgos al exámen físico & $\mathrm{N}$ & $\%$ \\
\hline Ictericia & 39 & 67.2 \\
Sangrado & 21 & 36.2 \\
Piel (76\%), & & \\
Subconjutival (24\%) & 16 & 27.5 \\
Hepatomegalia & 10 & 17.2 \\
Esplenomegalia & 6 & 10.3 \\
Rigidez nucal & \\
\hline
\end{tabular}

\begin{tabular}{|lc|}
\hline \multicolumn{2}{|c|}{$\begin{array}{c}\text { Cuadro 5:Alteraciones de laboratorio } \\
\text { asociados con leptospirosis }\end{array}$} \\
\hline Estudio & $\mathrm{N}$ \\
\hline Plaquetopenia & 40 \\
Pruebas de función renal alteradas & 40 \\
Leucopenia & 39 \\
Hiperbilirrubinemia directa & 39 \\
AST aumentada & 39 \\
CPK aumentado & 32 \\
Función renal alterada & 20 \\
Alteración de pruebas de coagulación & 20 \\
Hiponatremia & 18 \\
Leucocitocis & 12 \\
\hline
\end{tabular}

30 años están más propensas a realizar trabajos relacionados con labores agrícolas o de ganadería, las que suponen algún riesgo para contraer leptospirosis.

Debido a la inexistencia de investigaciones que involucren los tres cantones que componen esta región, es imposible hacer una comparación válida con los resultados obtenidos sobre procedencia; sin embargo, estos podrían atribuirse a dos factores: primero, a que los cantones de Guatuso y Los Chiles tienen una población dispersa, que habita en sitios alejados, en donde la cobertura de los medios de salud es menor, en consecuencia, una infección que se manifiesta en forma leve puede pasar desapercibida; segundo, a que el cantón de San Carlos tiene más población (según el Instituto Nacional de Estadística y Censos) y, por esta razón, se puede esperar que el mayor número de pacientes con leptospirosis habiten en esta área de la región.

En el presente estudio se determinó que la mayoría de los casos en la Región Huetar Norte se presentaron en el tercer trimestre del año, lo que coincide con el inicio de la estación lluviosa, favoreciendo la supervivencia y reproducción de las leptospiras en el ambiente, tal como lo cita la bibliografía ${ }^{1,23}$. Esta característica se mantuvo constante 
durante el periodo en estudio, con excepción del 2000, año cuando la incidencia fue mayor en el primer trimestre; sin embargo, debido a la pobre definición de las estaciones climáticas en Costa Rica y, sobre todo, en esta región, este no es un dato muy confiable.

Según el serotipo, los datos encontrados no coinciden con la información suministrada por el Ministerio de Salud, dado que, en el ámbito nacional, el serotipo más frecuente es el icteroaemorrhagie, el pyrogenes ocupa el cuarto lugar, mientras que el sejroe está en séptimo ${ }^{8}$. Los otros dos serotipos, tarassovi y hebdomadis, ni siquiera aparecen en la lista de frecuencia, pero sí se encuentran entre los más comunes en otros países como Estados Unidos y Turquía ${ }^{2,24}$.

Las manifestaciones clínicas y de laboratorio típicas de la leptospirosis ${ }^{1,2,3}$, fueron las mismas encontradas en este grupo de pacientes. Sin embargo, a pesar de que en la mayoría de los documentos revisados señala una característica y ligera leucocitosis, en esta investigación el hallazgo de leucopenia es tres veces más frecuente que el de leucocitosis, incluso en los casos en los que el diagnóstico se hizo en forma temprana. Basándose en este dato, se pudo conocer que existe un comportamiento clínico distinto al descrito en la literatura, de hecho, en la experiencia de los clínicos de la región, el diagnóstico está orientado a buscar los síntomas, signos y alteraciones de laboratorio descritos con anterioridad, más leucopenia.

La presencia de ictericia en más del $65 \%$ de los enfermos es otro resultado contrastante con la bibliografía, ya que en esta se afirma que la forma ictérica se presenta en menos del $10 \%$ de los casos de leptospirosis ${ }^{1,2,10}$. Existen al menos tres razones que podrían explicar esta diferencia, primero, es posible que haya subjetividad al momento de definir ictericia, ya que muchos médicos la describen como un tinte amarillento de piel y mucosas, y otros se basan en los hallazgos de laboratorio. Segundo, en la mayoría de la población de la zona la tez es morena y en ellas, por constitución, existe un leve tinte amarillo de las escleras, por último, la inespecificidad de las manifestaciones de la enfermedad, principalmente de la forma anictérica, sugieren pensar en una infección viral, lo que puede confundir el diagnóstico.

El hallazgo de factores de riesgo en casi la totalidad de los pacientes coincide con la mayoría de las investigaciones revisadas, en las que se recalca la importancia del uso de medidas preventivas para la disminución de la incidencia de esta enfermedad ${ }^{2,6,22}$.

Es importante contemplar la posibilidad de diseñar una campaña local de educación y concienciación a la población general y a los profesionales de la salud, en la que se expliquen las manifestaciones de la enfermedad, la forma de transmisión, así como también la manera en que se puede prevenir el contagio. Además, este proyecto debe hacer énfasis en la necesidad del uso de equipo de protección en las personas que tiene trabajos de alto riesgo, todo esto con el fin de disminuir la incidencia de leptospirosis en la zona y de lograr la detección temprana de la enfermedad.

\section{Abstract}

Aim and objectives: To determine the epidemiological behavior of leptospirosis among the population of the northern region (Región Huetar Norte) of Costa Rica, during the 4 year period comprised between 1997 to 2000 according to biomedical data issued by the regional headquarters of the Ministry of Health and the local Hospital.

Methods: A list of cases with positive serology for leptospirosis seen in the northern region from 1997 to the year 2000, was supplied by the INCIENSA database. Their medical records were obtained from the office of biostatistics of the San Carlos Hospital. The information was collected in a previously designed form. The results were analyzed with the Statistical Package for the Social Sciences (SPSS).

Results: 58 patients had positive serology for leptospirosis. Among them the male gender predominated in a 13:1 ratio. Young adults (ages from 15 to 30 years) were more frequently affected. Most of the people diagnosed with leptospirosis were natives of San Carlos. More than one third of the patients were engaged in agriculture or cattle handling. The serotypes more frequently detected were pomona followed by sejroe, tarassovi and hebdomadis.

The symptoms were fever, headaches, myalgia and bleeding. Laboratory findings renal and hepatic function tests abnormalities, thrombocytopenia and increased CPK were.

Conclusions: Risk factors were present in almost all of the patients. Occupational factors predominated. Leucopoenia was 3 times more frequent than leucocytosis. This fact would lead us to state that there is a clinical behavior of leptospirosis that differs from what is described in the literature.

\section{Referencias}

1. Mandell G, Bennet J, Dollin R. Enfermedades infecciosas. Principios y práctica. $4^{\mathrm{a}}$ ed. Buenos Aires. Editorial médica Panamericana S.A.; 1997

2. Lomar AV, Diament D, Torres JR. Leptospirosis in Latin America. Infect Dis Clin North Am 2000; 14: 23-39.

3. Plank R, Dean D. Overview of the epidemiology, microbiology, and pathogenesis of Leptospira ssp in humans. Microbes Infect 2000; 2: $1265-1276$

4. Clark C. Dengue-based system detects increased leptospirosis. World Disease Weekly 1999; 45:89-98. 
5. Centers for Disease Control and Prevention. Leptospirosis and unexplained acute febrile illness, 1998. JAMA, 1998; 280:1474-90.

6. Ko A, Galvao M, Ribeiro C, Jonson W, Riley L. Urban epidemic of severe leptospirosis in Brazil. Lancet 1999; 354: 820-32.

7. Rowland B. Gale Encyclopedia of Medicine. $1^{\mathrm{a}}$ ed. Gale Group Editorial; 2000.

8. Ministerio de Salud de Costa Rica. Protocolo para la prevención y control de la leptospirosis. Julio, 2000.

9. Organización Panamericana de la Salud. El control de las enfermedades transmisibles en el hombre. (16 ${ }^{\mathrm{a}}$ ed.) Washington: Editorial de la Organización Panamericana de la Salud; 1997.

10. Noone J. Diagnosis and treatment of leptospirosis in primary care setting. Nurse Pract 1998; 23:62-70.

11. Leblebiciog W. Weil's disease: report of 12 cases. JAMA 1999; 277:1744-52.

12. Browsher B, Callahan C, Person D, Ruess L. Unilateral leptospiral pneumoniae and cold agglutinin disease. Chest 1999 ; 116:830-41.

13. Clement J, Neild G, Lemos S, Crescente J, Ranst M. Urban leptospirosis versus urban hantavirus infection in Brazil. Lancet 1999 354:2003-15.

14. Ribeiro M, Souza C, Almeida S. Dot-ELISA for human leptospirosis employing inmunodominant antigen. J Trop Med Hy 1995; 98: 452-6.

15. Brown P, Gravecamp C, Carrinton D. Evaluation of the Polymerase Chain Reaction for early detection of leptospirosis. J Med Microb 1995; 43:110-14.
16. Merien F, Baranton G, Perolat P. Comparison of polymerase Chain reaction with MAT and culture for diagnosis of Leptospirosis. J Infec Dis 1995; 172:281-85.

17. Gravecamp C, Hartokeerl R. Detection of leptospires in urine by PCR for early detection of leptospirosis. J Clin Microb 1994; 32:1894-98.

18. Vinetz J, Glass G, Flexner C. Sporadic urban leptospirosis. Ann Int Med 1996; 125.794-98.

19. Gravecamp C. Mabs suitable for ELISA used for detection of L. interrogans. World Disease Weekly 2000; 26:77-83.

20. Takafuji E, Kirkpatrick J, Miller Z. An efficacy trial of doxyciline chemoprophylaxis against leptospirosis. N Engl J Med 1984; 310:497-500.

21. León E. Apuntes de Epidemiología Básica. Primera Edición. San José, Costa Rica: Litografía IPECA S.A.; 1999.

22. Centers for Disease Control and Prevention. Outbreak of leptospirosis among white-water rafters in Costa Rica. JAMA 1997; 278:808-12.

23. Fauci AS, Braunwald E. Principios de Medicina Interna de Harrison. 14 ed. Madrid: Mc Graw Hill-Interamericana; 1998.

24. Swich WJ, Greer P, Ferebee T, Goldsmith C, Guarner J, Zaki S. Emerging pathogens. Tuberculosis and Airbone Disease Weekly $1998 ; 26: 45-60$ 